\title{
ON COHOMOLOGY AUTOMORPHISMS OF COMPLEX FLAG MANIFOLDS
}

\author{
MICHAEL HOFFMAN AND WILLIAM HOMER
}

\begin{abstract}
We conjecture a classification of the automorphisms of the rational cohomology ring of $U(n) / H$ for $H$ a closed connected subgroup of maximal rank in $U(n)$, and prove a partial result.
\end{abstract}

1. Introduction. For any sequence $n_{1}, n_{2}, \ldots, n_{l}$ of positive integers with sum $n$, let $F\left(n_{1}, \ldots, n_{l}\right)$ denote the homogeneous space $U(n) / H$, where $H$ is a subgroup of $U(n)$ conjugate to $U\left(n_{1}\right) \times U\left(n_{2}\right) \times \cdots \times U\left(n_{l}\right)$. Then $F\left(n_{1}, \ldots, n_{l}\right)$ can be given the structure of a complex manifold of complex dimension $\sum_{p<q} n_{p} n_{q}$. We can think of $F\left(n_{1}, \ldots, n_{l}\right)$ as the space of orthogonal decompositions

$$
\mathbf{C}^{n}=p_{1} \oplus p_{2} \oplus \cdots \oplus p_{l}
$$

with $\operatorname{dim} p_{i}=n_{i}$. Evidently there are canonical $n_{i}$-plane bundles over $F\left(n_{1}, \ldots, n_{l}\right)$ for $1 \leq i \leq l$ : we denote these bundles $\eta_{i}$ and set

$$
x_{i, h}=c_{h}\left(\eta_{i}\right) \in H^{2 h}\left(F\left(n_{1}, \ldots, n_{l}\right) ; \mathbf{Q}\right), \quad 1 \leq h \leq n_{i} .
$$

The structure of $H^{*}\left(F\left(n_{1}, \ldots, n_{l}\right) ; \mathbf{Q}\right)$ is given by the following result $[\mathbf{1}]$.

THEOREM 1.1. The cohomology ring $H^{*}\left(F\left(n_{1}, \ldots, n_{l}\right) ; \mathbf{Q}\right)$ is isomorphic to the quotient

$\mathbf{Q}\left[x_{i, h} \mid 1 \leq i \leq l, 1 \leq h \leq n_{i}\right] /\left(1+x_{1,1}+\cdots+x_{1, n_{1}}\right) \cdots\left(1+x_{l, 1}+\cdots+x_{l, n_{l}}\right)=1$.

We make the following conjecture (cf. [4]).

CONJECTURE 1.2. Any automorphism $\varphi$ of $H^{*}\left(F\left(n_{1}, \ldots, n_{l}\right)\right.$; Q) has the form $\varphi\left(x_{i, h}\right)=m^{h} x_{\sigma(i), h}$ where $m \neq 0$ and $\sigma$ is a permutation of $\{1,2, \ldots, l\}$ such that $n_{p}=n_{q}$ whenever $\sigma(p)=q$.

REMARKS. 1. We can assume $n_{1} \leq n_{2} \leq \cdots \leq n_{l}$.

2. Any $F\left(n_{1}, \ldots, n_{l}\right)$ for which Conjecture 1.2 holds has trivial genus [5].

Conjecture 1.2 has been proved in the following special cases:

1. $l=2[\mathbf{2}, \mathbf{6}]$;

2. $l=3, n_{2}>n_{1}=1$ and $n_{3} \geq 2 n_{2}^{2}-1[4]$;

3. $n_{1}=n_{2}=\cdots=n_{l-1}=1[3]$.

In this paper we provide further evidence for Conjecture 1.2 by proving that any automorphism of $H^{*}\left(F\left(n_{1}, \ldots, n_{l}\right) ; \mathbf{Q}\right)$ acts on $H^{2}\left(F\left(n_{1}, \ldots, n_{l}\right) ; \mathbf{Q}\right)$ as expected (Theorem 3.2); this implies a partial result for all dimensions (Corollary 3.3). The proof is based on results of $\S 2$, where the height in $H^{*}\left(F\left(n_{1}, \ldots, n_{l}\right)\right.$; $\left.\mathbf{Q}\right)$ of any two-dimensional cohomology class is determined.

The authors thank Allen Broughton for prodding them into proving Theorem 2.3.

Received by the editors May 9, 1983 .

1980 Mathematics Subject Classification. Primary 55S37; Secondary 57T15.

Key words and phrases. Flag manifold, cohomology ring, automorphism.

(C) 1984 American Mathematical Society $0002-9939 / 84 \$ 1.00+\$ .25$ per page 
2. Heights of two-dimensional cohomology classes. Let $1^{n}$ denote 1 repeated $n$ times. Then $H^{*}\left(F\left(1^{n}\right) ; \mathbf{Q}\right)$ is generated by two-dimensional elements, which we denote by $t_{i}$ (instead of $x_{i, 1}$ as above). By Theorem $1.1, H^{*}\left(F\left(1^{n}\right)\right.$; $\mathbf{Q}$ ) is the polynomial algebra $\mathbf{Q}\left[t_{1}, \ldots, t_{n}\right]$ modulo the ideal generated by the elementary symmetric functions $e_{i}\left(t_{1}, \ldots, t_{n}\right)$.

We denote the symmetric group on $\{1,2, \ldots, n\}$ by $\Sigma_{n}$. Let $P=\left\{P_{1}, P_{2}, \ldots, P_{l}\right\}$ be a partition of $\{1,2, \ldots, n\}$, and put

$$
\Sigma_{P}=\left\{\sigma \in \Sigma_{n} \mid \sigma(p)=q \text { implies } p, q \in P_{k}, \text { some } k\right\} .
$$

If card $P_{i}=n_{i}$, there is a map $\pi_{P}: F\left(1^{n}\right) \rightarrow F\left(n_{1}, \ldots, n_{l}\right)$ given by

$$
\pi_{P}\left(l_{1}, l_{2}, \ldots, l_{n}\right)=\left(p_{1}, p_{2}, \ldots, p_{l}\right), \quad p_{i}=\bigoplus_{k \in P_{i}} l_{k} .
$$

The symmetric group $\Sigma_{n}$ acts on $H^{*}\left(F\left(1^{n}\right) ; \mathbf{Q}\right)$ by permuting the $t_{i}$. For any subgroup $G$ of $\Sigma_{n}$, let $H^{*}\left(F\left(1^{n}\right) ; \mathbf{Q}\right)^{G}$ be the subring invariant under $G$. The next result shows that any cohomology ring $H^{*}\left(F\left(n_{1}, \ldots, n_{l}\right) ; \mathbf{Q}\right)$ with $n_{1}+\cdots+n_{l}=n$ can be regarded as an invariant subring of $H^{*}\left(F\left(1^{n}\right) ; \mathbf{Q}\right)$.

Proposition 2.1. For any partition $P=\left\{P_{1}, \ldots, P_{l}\right\}$ of $\{1,2, \ldots, n\}$ with card $P_{i}=n_{i}$, the map $\pi_{P}^{*}: H^{*}\left(F\left(n_{1}, \ldots, n_{l}\right) ; \mathbf{Q}\right) \rightarrow H^{*}\left(F\left(1^{n}\right) ; \mathbf{Q}\right)$ is an injection with image $H^{*}\left(F\left(1^{n}\right) ; \mathbf{Q}\right)^{\Sigma_{P}}$.

PROOF. There is a fibration

$$
F\left(1^{n_{1}}\right) \times F\left(1^{n_{2}}\right) \times \cdots \times F\left(1^{n_{l}}\right) \rightarrow F\left(1^{n}\right) \stackrel{\pi_{P}}{\rightarrow} F\left(n_{1}, \ldots, n_{l}\right),
$$

whose Serre spectral sequence collapses for degree reasons, so $\pi_{P}^{*}$ injects. Now $\pi_{P}^{*}$ maps $x_{i, h} \in H^{*}\left(F\left(n_{1}, \ldots, n_{l}\right) ; \mathbf{Q}\right)$ to $e_{h}\left(P_{i}\right)$, the $h$ th elementary symmetric function in the variables $\left\{t_{k} \mid k \in P_{i}\right\}$. Since $\Sigma_{P}=\Sigma\left(P_{1}\right) \times \Sigma\left(P_{2}\right) \times \cdots \times \Sigma\left(P_{l}\right)$, where $\Sigma\left(P_{i}\right)$ is the symmetric group on $P_{i}$, the conclusion follows.

For any homogeneous element $u$ of $H^{*}\left(F\left(n_{1}, \ldots, n_{l}\right) ; \mathbf{Q}\right)$, we define the height of $u$ to be the greatest power $p$ so that $u^{p} \neq 0$. The next result gives an upper bound on height $u$ for $u \in H^{2}\left(F\left(n_{1}, \ldots, n_{l}\right) ; \mathbf{Q}\right)$. (This upper bound will turn out be exactly height $u$, as we show in Theorem 2.3.)

THEOREM 2.2. Let

$$
u=a_{1} x_{1,1}+a_{2} x_{2,1}+\cdots+a_{l} x_{l, 1} \in H^{2}\left(F\left(n_{1}, \ldots, n_{l}\right) ; \mathbf{Q}\right) .
$$

Define an equivalence relation $E$ on $\{1,2, \ldots, l\}$ by $E=\left\{(p, q) \mid a_{p}=a_{q}\right\}$, and let $P(E)=\left\{S_{1}, S_{2}, \ldots, S_{r}\right\}$ be the set of equivalence classes. Then

$$
\text { height } u \leq \sum_{p<q} m_{p} m_{q}
$$

where

$$
m_{i}=\sum_{k \in S_{i}} n_{k}, \quad 1 \leq i \leq r .
$$

Proof. Choose a partition $P=\left\{P_{1}, \ldots, P_{l}\right\}$ of $\{1,2, \ldots, n\}$ with $\operatorname{card} P_{i}=n_{i}$. By the preceding result, we can identify $H^{*}\left(F\left(n_{1}, \ldots, n_{l}\right) ; \mathbf{Q}\right)$ with $H^{*}\left(F\left(1^{n}\right) ; \mathbf{Q}\right)^{\Sigma_{P}}$. 
Then $u \in H^{2}\left(F\left(1^{n}\right)\right.$; $\left.\mathbf{Q}\right)$ has isotropy subgroup $\Sigma_{Q}$, where $Q=\left\{Q_{1}, \ldots, Q_{r}\right\}, Q_{i}=$ $\bigcup\left\{P_{k} \mid k \in S_{i}\right\}$. Since card $Q_{i}=m_{i}$, we have

$$
u \in H^{*}\left(F\left(1^{n}\right) ; \mathbf{Q}\right)^{\Sigma_{Q}} \cong H^{*}\left(F\left(m_{1}, \ldots, m_{r}\right) ; \mathbf{Q}\right)
$$

and (1) follows since the right-hand side is the complex dimension of $F\left(m_{1}, \ldots, m_{r}\right)$.

Now let $u$ be as in the statement of the preceding result, and define a polynomial $f$ in the $a_{i}$ by

$$
f\left(a_{1}, a_{2}, \ldots, a_{l}\right)=\left\langle u^{d},\left[F\left(n_{1}, \ldots, n_{l}\right)\right]\right\rangle
$$

where $d=\sum_{p<q} n_{p} n_{q}$ and $\langle$,$\rangle denotes Kronecker product. We say u$ has maximal height in $H^{*}\left(F\left(n_{1}, \ldots, n_{l}\right) ; \mathbf{Q}\right)$ if $u^{d} \neq 0$.

THEOREM 2.3. There is a constant $K \neq 0$ so that

$$
f\left(a_{1}, \ldots, a_{l}\right)=K \prod_{p<q}\left(a_{p}-a_{q}\right)^{n_{p} n_{q}} .
$$

In particular, $u$ has maximal height in $H^{*}\left(F\left(n_{1}, \ldots, n_{l}\right)\right.$; $\left.\mathbf{Q}\right)$ if and only if all the $a_{i}$ are distinct. It follows that (1) of Theorem 2.2 is an equality.

PROOF. For $1 \leq p<q \leq l$, let

$$
D_{p q}=\frac{\partial}{\partial\left(a_{p}-a_{q}\right)}=\frac{1}{2}\left(\frac{\partial}{\partial a_{p}}-\frac{\partial}{\partial a_{q}}\right) .
$$

Then $D_{p q} u=\left(x_{p, 1}-x_{q, 1}\right) / 2$. Apply $D_{p q}^{r}$ to both sides of

$$
f\left(a_{1}, \ldots, a_{l}\right)=\left\langle u^{d},\left[F\left(n_{1}, \ldots, n_{l}\right)\right]\right\rangle
$$

to get

$$
D_{p q}^{r} f=\left\langle d(d-1) \cdots(d-r+1) u^{d-r}\left(D_{p q} u\right)^{r},\left[F\left(n_{1}, \ldots, n_{l}\right)\right]\right\rangle .
$$

For $r<n_{p} n_{q}$ we have $u^{d-r}=0$ when $a_{p}=a_{q}$, by 2.2. Thus $\left(a_{p}-a_{q}\right)^{n_{p} n_{q}}$ divides $f\left(a_{1}, \ldots, a_{l}\right)$ for every $p<q$. Then either $(2)$ holds or $f\left(a_{1}, \ldots, a_{l}\right)$ is identically zero. But $F\left(n_{1}, \ldots, n_{l}\right)$ can be given the structure of a Kähler manifold [3], so there must be some two-dimensional class of maximal height: thus, (2) holds. Then (1) must be an equality since the hypothesis of 2.2 implies that $u$ has maximal height in $H^{*}\left(F\left(m_{1}, \ldots, m_{r}\right)\right.$; $\left.\mathbf{Q}\right)$.

REMARK. A different proof of the formula for height $u$ is given in [3].

3. Proof of the main theorem. To prove the main theorem we require a combinatorial lemma.

LEMMA 3.1. Let $S \subset\{(p, q) \mid 1 \leq p<q \leq l\}, \bar{S}$ the equivalence relation generated by $S$, and $P(\bar{S})$ the partition of $\{1,2, \ldots, l\}$ into $\bar{S}$-equivalence classes. If $\operatorname{card} S=\left(\begin{array}{c}l-1 \\ 2\end{array}\right)$, then

1. $P(\bar{S})=\{\{1,2, \ldots, l\}\}$, or

2. $P(\bar{S})=\{\{1,2, \ldots, k-1, k+1, \ldots, l\},\{k\}\}$ for some $1 \leq k \leq l$.

Proof. Let $P(\bar{S})=\left\{P_{1}, P_{2}, \ldots, P_{t}\right\}$. Then

$$
\operatorname{card} S \leq \sum_{i=1}^{t}\left(\begin{array}{c}
\operatorname{card} P_{i} \\
2
\end{array}\right)=\left(\begin{array}{l}
l \\
2
\end{array}\right)-\sum_{p<q} \operatorname{card} P_{p} \operatorname{card} P_{q} .
$$


By the hypothesis, this implies

$$
\sum_{p<q} \operatorname{card} P_{p} \operatorname{card} P_{q} \leq l-1
$$

which can happen only if (1) or (2) holds.

Now we prove the main result.

THEOREM 3.2. Let $\varphi$ be an automorphism of $H^{*}\left(F\left(n_{1}, \ldots, n_{l}\right) ; \mathbf{Q}\right)$. Then

$$
\varphi\left(x_{i, 1}\right)=m x_{\sigma(i), 1}, \quad 1 \leq i \leq l,
$$

where $m \neq 0$ and $\sigma$ is a permutation of $\{1,2, \ldots, l\}$ such that $n_{p}=n_{q}$ whenever $\sigma(p)=q$.

ProOF. Let $V$ denote the quotient of the free rational vector space on $x_{1,1}, x_{2,1}$, $\ldots, x_{l, 1}$ by $\mathbf{Q}\left(x_{1,1}+x_{2,1}+\cdots+x_{l, 1}\right)$. We identify $V$ with $H^{2}\left(F\left(n_{1}, \ldots, n_{l}\right) ; \mathbf{Q}\right)$, so $\varphi$ is a linear automorphism of $V$ which preserves heights. For $1 \leq p<q \leq l$, put

$$
H_{p q}=\left\{a_{1} x_{1,1}+\cdots+a_{l} x_{l, 1} \in V \mid a_{p}=a_{q}\right\} .
$$

Then each $H_{p q}$ is a hyperplane in $V$, and

$$
\bigcap_{p<q}\left(V-H_{p q}\right)=V-\bigcup_{p<q} H_{p q}
$$

is precisely the set of vectors of maximal height. It follows that $\varphi$ must permute the $H_{p q}$.

Let $S \subset\{(p, q) \mid 1 \leq p<q \leq l\}$. It is easy to see that

$$
\bigcap_{(p, q) \in S} H_{p q}=\mathbf{Q}\left(x_{1,1}+\cdots+x_{l, 1}\right)=\{0\}
$$

if and only if $P(\bar{S})=\{\{1,2, \ldots, l\}\}$, in the notation of the lemma. Now if $1 \leq i \leq l$,

$$
\mathbf{Q} x_{i, 1}=\bigcap_{\substack{p<q \\ p, q \neq i}} H_{p q} \text {. }
$$

Since $\varphi$ permutes the $H_{p q}$, we have

$$
\varphi\left(\mathbf{Q} x_{i, 1}\right)=\bigcap_{(p, q) \in S} H_{p q}
$$

for some $S$ with card $S=\left(\begin{array}{c}l-1 \\ 2\end{array}\right)$. By the lemma, either

1. $P(\bar{S})=\{\{1,2, \ldots, l\}\}$, or

2. $P(\bar{S})=\{\{1,2, \ldots, k-1, k+1, \ldots, l\},\{k\}\}$ for some $1 \leq k \leq l$.

But (1) cannot hold, since $\varphi$ cannot take a line to $\{0\}$. Thus (2) holds, and

$$
\varphi\left(\mathbf{Q} x_{i, 1}\right)=\bigcap_{\substack{p<q \\ p, q \neq k}} H_{p q}=\mathbf{Q} x_{k, 1} .
$$

It follows that

$$
\varphi\left(x_{i, 1}\right)=m_{i} x_{\sigma(i), 1}, \quad 1 \leq i \leq l,
$$

for some permutation $\sigma$ of $\{1,2, \ldots, l\}$. Then

$$
0=\varphi\left(x_{1,1}+\cdots+x_{l, 1}\right)=m_{1} x_{\sigma(1), 1}+\cdots+m_{l} x_{\sigma(l), 1},
$$


so $m_{1}=m_{2}=\cdots=m_{l}=m$ (clearly $\left.m \neq 0\right)$.

Finally, suppose $\sigma(p)=q$. Then

$$
n_{p}\left(n-n_{p}\right)=\text { height } x_{p, 1}=\text { height } x_{q, 1}=n_{q}\left(n-n_{q}\right),
$$

where we use the height formula. Since

$$
n_{p}\left(n-n_{p}\right)-n_{q}\left(n-n_{q}\right)=\sum_{k \neq p, q}\left(n_{p}-n_{q}\right) n_{k},
$$

we have $n_{p}=n_{q}$ (unless $l=2$, in which case the theorem is trivially true).

REMARK. In the preceding result it suffices to assume $\varphi$ is an endomorphism of $H^{*}\left(F\left(n_{1}, \ldots, n_{l}\right)\right.$; $\left.\mathbf{Q}\right)$ which is an automorphism on dimension 2. For if $\varphi$ is an automorphism of $H^{2}\left(F\left(n_{1}, \ldots, n_{l}\right) ; \mathbf{Q}\right)$, it must take some class of maximal height to another class of maximal height (since the preimage of a class of maximal height has maximal height). Hence $\varphi$ is nonzero on a generator of

$$
H^{2 d}\left(F\left(n_{1}, \ldots, n_{l}\right) ; \mathbf{Q}\right), \quad d=\sum_{p<q} n_{p} n_{q} .
$$

Then consideration of Poincaré duality shows that $\varphi$ is an automorphism of $H^{*}\left(F\left(n_{1}, \ldots, n_{l}\right) ; \mathbf{Q}\right)$.

Theorem 3.2 yields the following result in higher dimensions.

Corollary 3.3. Let $D$ be the decomposables of $H^{2 h}\left(F\left(n_{1}, \ldots, n_{l}\right) ; \mathbf{Q}\right)$, i.e. sums of products of positive-dimensional elements. Then for any automorphism $\varphi$ of $H^{*}\left(F\left(n_{1}, \ldots, n_{l}\right) ; \mathbf{Q}\right)$ and $i$ with $n_{i} \geq h$,

$$
\varphi\left(x_{i, h}\right)=m_{h} x_{\sigma(i), h} \bmod D
$$

where $m_{h} \neq 0$ and $\sigma$ is a permutation of $\left\{i \mid n_{i} \geq h\right\}$ such that $\left[n_{p} / h\right]=\left[n_{q} / h\right]$ whenever $\sigma(p)=q$.

Proof. Let $\bar{D}$ be the ideal in $H^{*}\left(F\left(n_{1}, \ldots, n_{l}\right)\right.$; Q $)$ generated by $\left\{x_{i, k} \mid 1 \leq\right.$ $i \leq l$ and $k$ does not divide $h\}$. Then $\varphi(\bar{D}) \subset \bar{D}$, so $\varphi$ induces an endomorphism $\bar{\varphi}$ of $H^{*}\left(F\left(n_{1}, \ldots, n_{l}\right) ; \mathbf{Q}\right) / \bar{D}$. In fact $\bar{\varphi}$ is an automorphism, since it has inverse $\overline{\varphi^{-1}}$. Now there is an isomorphism

$$
H^{*}\left(F\left(n_{1}, \ldots, n_{l}\right) ; \mathbf{Q}\right) / \bar{D} \stackrel{\sim}{\rightarrow} H^{* h}\left(F\left(\left[n_{1} / h\right], \ldots,\left[n_{l} / h\right]\right) ; \mathbf{Q}\right),
$$

with the indicated shift in dimension. Apply 3.2 to $\bar{\varphi}$ to get the conclusion (note $\left.H^{2 h}\left(F\left(n_{1}, \ldots, n_{l}\right) ; \mathbf{Q}\right) \cap \bar{D}=D\right)$.

REMARK. If $n_{p} \geq 2 n_{p-1}$ for each $p$, it follows that $\varphi\left(x_{i, h}\right)=m_{h} x_{i, h} \bmod$ decomposables for all $i$ and $h$.

\section{REFERENCES}

1. A. Borel, Sur la cohomologie des espaces fibrés principaux et des espaces homogénes de groupes de Lie compacts, Ann. of Math. (2) 57 (1953), 115-207.

2. S. Brewster and W. Homer, Rational automorphisms of Grassmann manifolds, Proc. Amer. Math. Soc. 88 (1983), 181-183.

3. S. A. Broughton, M. Hoffman and W. Homer, The height of two-dimensional cohomology classes of complex flag manifolds, Canad. Math. Bull. 26 (1983), 498-502.

4. H. Glover and W. Homer, Self maps of flag manifolds, Trans. Amer. Math. Soc. 267 (1980), 423-434. 
5. H. Glover and G. Mislin, On the genus of generalized flag manifolds, Enseignment Math. 27 (1981), 211-219.

6. M. Hoffman, Endomorphisms of the cohomology of complex Grassmannians, Trans. Amer. Math. Soc. 281 (1984), 745-760.

Department of Mathematics, Ohio State University, Columbus, Ohio 43210 (Current address of Michael Hoffman)

Department of Mathematics, Memorial University of Newfoundland, St. JOHN'S, NEWFOUNDLAND, CANADA A1B 3X7

Current address (William Homer): Intermetrics, Inc., Cambridge, Massachusetts 